\title{
A LOGICAL APPROACH TO ARABIC PHONOLOGY
}

\author{
Steven Bird \& Patrick Blackburn \\ University of Edinburgh, Centre for Cognitive Science \\ 2 Buccleuch Place, Edinburgh EH8 9LW, Scotland \\ stevenecogsci.ed.ac.uk, patrickecogsci.ed.ac.uk
}

\begin{abstract}
Logical approaches to linguistic description, particularly those which employ feature structures, have generally treated phonology as though it was the same as orthography. This approach breaks down for languages where the phonological shape of a morpheme can be heavily dependent on the phonological shape of another, as is the case in Arabic. In this paper we show how the tense logical approach investigated by Blackburn (1989) can be used to encode hierarchical and temporal phonological information of the kind explored by Bird (1990). Then we show how some Arabic morphemes may be represented and combined ${ }^{1}$.
\end{abstract}

\section{INTRODUCTION}

There is an increasingly widespread view that linguistic behaviour results from the complex interaction of multiple sources of partial information. This is exemplified by the rapidly growing body of work on natural language syntax and semantics such as the Unification-Based Grammar Formalisms. Similarly, in phonology there is a popular view of phonological representations as having the same topology as a spiral-bound notebook, where segments (or slots) are strung out along the spine and each page gives a structural description of that string according to some descriptive vocabulary. Crucially only those segmental strings which are licensed by all of the independent descriptions are acceptable.

The practical difficulty is to come up with a model of grammatical organization which allows the right information to be brought to bear at the right stage. One model which looks particularly attractive in this regard considers the traditional modules of grammar (i.e. syntax, semantics and phonology) not in series where the output of one feeds into the input of the next, but rather in parallel, where each module exerts independent constraints. For example, a morpheme may be represented as a complex consisting of a semantic expression, a constraint on (morpho)syntactic distribution, and a phonological description. The combination of morphemes is then subject to three independent calculi, one per domain. The result is a compositional account of the relationship between form and meaning, as evidenced in the work of Bach (1983), Hoeksema \& Janda (1988) and Wheeler (1988). However, all these proposals have assumed that phonological representations are merely linear

\footnotetext{
1 We are indebted to Mike Reape, Ewan Klein and members of the Edinburgh Applied Logic group for discussions of the material presented here. We are grateful for the support of ESPRIT Basic Research Action 3175 (DYANA) and SERC post-doctoral fellowship B/ITF/255.
}

sequences of segments. Such a restriction renders a theory incapable of expressing the observations which have been made in the non-linear phonology literature (e.g. Goldsmith 1990). Bird \& Klein (1990) and Bird (1990) have endeavoured to show how the compositional approach can be liberated from a purely linear segmental view of phonology. This paper exemplifies and extends those proposals.

The first section presents a logical language for phonological description. The second section shows how it has sufficient expressive power to encompass a variety of observations about syllable structure. The final section discusses further observations which can be made about Arabic syllable structure, and provides an illustrative treatment of socalled non-concatenative morphology in the perfect tense verb paradigm.

\section{LOGICAL FRAMEWORK}

Interval based tense logics are calculi of temporal reasoning in which propositions are assigned truth values over extended periods of time ${ }^{2}$. Three operators $F$ (future), $P$ (past) and $O$ (overlaps) are introduced: $F \phi$ means " $\phi$ will be the case (at least once)", $P \phi$ means " $\phi$ was the case (at least once)" and $O \phi$ means " $\phi$ is the case at some overlapping interval (at least once)". $O$ corresponds to what phonologists call 'association'. Typically sentences are true at some intervals and not at others. (This is obviously the case, for example, if $\phi$ encodes the proposition "the sun is shining".) Blackburn (1989) has explored the effects of adding of a new type of symbol, called nominals, to tense logic. Unlike ordinary propositions, nominals are only ever true once. In a sense, a nominal is a 'name' (or a 'temporal indexical') for that unique period of time at which it is true. Certain observations about time can only be expressed in a theory which employs nominals. For example, $i \rightarrow \neg F i$ picks out precisely the irreflexive time flows, whereas no formula containing only propositional variables can do this. Nominals have been employed in the analysis of temporal reference in linguistic semantics. The present paper illustrates an application of nominals to a very different domain, namely phonology. In addition to $F, P$ and $O$, we shall employ the modality $\diamond$ to represent phonological dominance ${ }^{3}$.

\footnotetext{
2 See van Benthem 1983 for an introduction to this field and a survey of a variety of possible formulations of temporal structure.

3 The notion of dominance as employed in phonology corresponds closely to the use of the term in feature logics. However we treat dominance as a relation rather than as a collection of partial functions, for two reasons. First, in phonological structures, it is the nodes and not the arcs which are labelled. Second, there can be multiple arcs emanating from a node.
} 
Syntax. Let $X=\{p, q, r, \ldots\}$ be the propositional variables and let $N=\{i, j, k, \ldots\}$ be the nominals of $L$. Then $\mathcal{L}$ is the smallest set such that all of the following hold, where $\phi, \psi \in \mathcal{L}$.

$$
\begin{array}{ll}
T, \perp \in \mathcal{L} & X, N \subset \mathcal{L} \\
\diamond \phi, F \phi, P \phi, O \phi \in \mathcal{L} & \phi \vee \psi, \neg \phi \in \mathcal{L}
\end{array}
$$

We define $\rightarrow, \leftrightarrow$ and $\wedge$ as usual. We also define the duals of our modal operators in the usual fashion: $G \phi \equiv \neg F \neg \phi$ ( $\phi$ is always going to be the case), $H \phi \equiv \neg P \neg \phi$ ( $\phi$ always has been the case), $C \phi \equiv \neg O \neg \phi$ ( $\phi$ holds at all overlapping intervals) and $\square \phi \equiv \neg \diamond \neg \phi$ ( $\phi$ is true at all 'daughter' intervals). Two additional defined operators will play an important role in what follows: $M \phi \equiv P \phi \vee O \phi \vee F \phi$, and its dual $L \phi \equiv \neg M \neg \phi$. It follows from the semi-linear time semantics adopted below that $M \phi$ means ' $\phi$ holds at some time' and $L \phi$ means ' $\phi$ holds at all times'. We will often abbreviate $\diamond(p \wedge \phi)$ using the expression $(p) \phi$ and abbreviate a sequence of such applications $\left(p_{1}\right\rangle \cdots\left\langle p_{n}\right) \phi$ using the expression $\left\langle p_{1} \cdots p_{n}\right\rangle \phi$. We adopt a similar practice for the dual forms: [p] $\phi$ is shorthand for $\square(p \rightarrow \phi)$ and $\left[p_{1} \cdots p_{n}\right] \phi$ is shorthand for $\left[p_{1}\right] \cdots\left[p_{n}\right] \phi$. We also write $\diamond^{n}$ (or $\square^{n}$ ) to stand for a length $n$ sequence of $\rangle_{s}$ (or $\square s$ ).

Semantics. Let $T$ be a set of intervals (which we will think of as nodes), and let $\delta,<$ and o be binary relations on $T$. As $<$ models temporal precedence, it must be irreflexive and transitive. o models temporal overlap (phonological association), and so it is reflexive and symmetric. $<$ and 0 interact as follows: (i) they are disjoint, (ii) for any $t_{1}$, $t_{2}, t_{3}, t_{4} \in T, t_{1}<t_{2} \circ t_{3}<t_{4}$ implies $t_{1}<t_{4}$ (that is, precedence is transitive through overlap), and (iii) for any $t_{1}, t_{2} \in T, t_{1}<t_{2}$ or $t_{1} \circ t_{2}$ or $t_{1}>t_{2}$ (that is, our conception of time is semi-linear). Note that the triple $\langle T,<, 0\rangle$ is what temporal logicians call an interval structure.

The remaining relation $\delta$ encodes the hierarchical organization of phonological structures. As a phonological unit overlaps all of its constituents (cf. Hayes 1990:44), we demand that the transitive closure of $\delta$ be contained within o. Furthermore, phonological structures are never cyclic and so we require that for any $t_{1}, \ldots, t_{n} \in T$, if $t_{1} \delta t_{2}, \ldots$, $t_{n-1} \delta t_{n}$ then it is not the case that $t_{n} \delta t_{1}$. By a phonological frame $\mathcal{F}$ we mean a quadruple $(T,<, \circ, \delta)$ of the type just described.

It merely remains to link $L$ with such structures. A valuation $\nu$ is a function $(X \cup N) \rightarrow 2^{T}$ that obeys three constraints. First, it must assign a singleton set to each nominal. Second, for each $t \in T$, there is an $i \in N$ such that $\mathcal{V}(i)=\{t\}$. Third, if $t_{1}, t_{2} \in \mathcal{V}(p)$ where $p \in X$ then $t_{1} \circ t_{2} \rightarrow t_{1}=t_{2}$. In short, valuations are functions which ensure nominals act as names, where all intervals are named, and valuations capture the idea that phonological 'tiers' are linearly ordered. A model for $L$ is a pair $(\mathcal{F}, \mathcal{V})$. Satisfaction. Let $\mathcal{M}=\langle\mathcal{F}, \mathcal{V}\rangle, t \in T, a \in X \cup N$. Then:

$$
\begin{aligned}
& \mathcal{M} \vDash t, \quad \mathcal{M} \not t \perp \\
& \mathcal{M} \models_{t} a \text { iff } t \in \mathcal{V}(a) \quad \mathcal{M} \models t \neg \phi \text { iff } \mathcal{M} \not \neq_{t} \phi \\
& \mathcal{M} \models_{t} \phi \vee \psi \text { iff } \mathcal{M} F_{t} \phi \text { or } \mathcal{M} \models_{t} \psi \\
& \mathcal{M} \models t \delta_{\phi} \text { iff } \exists t^{\prime}: t \delta t^{\prime} \text { and } \mathcal{M} \models k_{t^{\prime}} \phi \\
& \mathcal{M} \vDash \models_{t} O \phi \text { iff } \exists t^{\prime}: t \circ t^{\prime} \text { and } \mathcal{M} \models t^{\prime} \phi \\
& \mathcal{M} \models, F \phi \text { iff } \exists t^{\prime}: t<t^{\prime} \text { and } \mathcal{M} \vDash \models^{\prime} \phi \\
& \mathcal{M} \models t P \phi \text { iff } \exists t^{\prime}: t^{\prime}<t \text { and } \mathcal{M} \equiv t^{\prime} \phi
\end{aligned}
$$

If $\mathscr{M} \models_{t} \phi$ then we say that $\phi$ is true in $\mathcal{M}$ at $t$. Note that under this semantics, $M$ really does mean 'at some time' and $L$ means 'at all times' (by virtue of semi-linearity).

Validities. If $\langle\mathcal{F}, \mathcal{V}\rangle \models_{t} \phi$ for all frames $\mathscr{F}$, for all valuations $\mathcal{V}$ on $\mathcal{F}$, and all $t \in T$. then we say $\phi$ is a validity. The following are some examples of validities. The first group concerns our intervalic structure.

(T1) $i \rightarrow \neg F i$. Precedence is irreflexive.

(T2) $\phi \rightarrow O \phi$. Overlap is reflexive.

(T3) $\phi \rightarrow C O \phi$. Overlap is symmetric.

(T4) $\mathrm{Fi} \rightarrow \neg \mathrm{Oi}_{i} \mathrm{Pi} \rightarrow \neg \mathrm{Oi}$. Precedence and overlap are disjoint.

(T5) $F O F \phi \rightarrow F \phi$. Precedence is transitive through overlap.

(T6) $F \phi \wedge F \psi \rightarrow F(\phi \wedge F \psi) \vee F(\psi \wedge F \phi) \vee F(\phi \wedge O \psi)$. Time is semi-linear".

The next two validities concern the dominance relation and its interaction with the interval structure.

(D1) $\diamond^{n} \phi \rightarrow O \phi$. The transitive closure of dominance is included in the overlap relation.

(D2) $i \rightarrow \neg \diamond^{n} i$. Dominance is acyclic.

The next group of validities reflect the constraints we have placed on valuations.

(FORCE) $M i$. Each nominal names at least one interval.

$(\mathrm{NOM}) i \wedge M(i \wedge \phi) \rightarrow \phi$.

Each nominal names at most one interval.

(PLIN) $p \wedge O(p \wedge \phi) \rightarrow \phi$.

Phonological liers are linearly ordered.

Proof Theory. It is straightforward using techniques discussed in (Gargov et al. 1987, 1989. Blackburn 1990) to provide a proof theory and obtain decidability results. At present we are investigating efficient proof methods for this logic and hope to implement a theorem prover.

\section{EXPRESSING PHONOLOGICAL CONSTRAINTS}

Feature Matrices. $\mathcal{L}$ can be used for describing feature matrices. For example, consider the matrix below.

[ PHON (Kay, pats, Blackle)]

A possible description of this matrix is: $\langle$ PHON)(Kay $\wedge$ $F i) \wedge\langle$ PHON $\rangle($ pats $\wedge i \wedge F j) \wedge\langle$ PHON $\rangle($ Blackie $\wedge j)$. This representation of sequences (cf. Rounds \& Manaster-Ramer 1987) enables the expression of partial ordering constraints which are widely required in phonological descriptions. Note that all instances of the following variant of the NOM schema are valid. $E$ and $E^{\prime}$ are strings of modal operators from $\{\diamond, F, P, O\}$.

$\left(N_{\mathcal{E}}\right) E_{i} \wedge E^{\prime}(i \wedge \phi) \leftrightarrow E(i \wedge \phi) \wedge E^{\prime} i$.

Formulas may be transferred between different palhs to the same interval.

${ }^{4}$ The mirror image of this formula, obtained by replacing all $F s$ with $P_{\mathrm{s}}$, is also valid. 
This schema expresses a familiar equivalence on feature matrices. For example:

$$
\left[\begin{array}{ll}
\text { SUBJ|AGR } & 1 \mathrm{pl} \\
\text { PRED|AGR } & 1
\end{array}\right] \equiv\left[\begin{array}{ll}
\text { SUBJ|AGR } & 1 \\
\text { PRED|AGR } & 1 \text { pl }
\end{array}\right]
$$

That is, nominals may be used in the representation of reentrancy (Bird 1991).

Sort Lattlces. Node labels in phonologists' diagrams (e.g. see example (1)) can be thought of as classifications. For example, we can think of $\mu \in X$ as denoting a certain class of nodes in a phonological structure (the mora nodes). Moras may be further classified into onset moras and coda moras, which are written as $\mu_{0}$ and $\mu_{c}$ respectively. The relationship between $\mu_{,} \mu_{o}$ and $\mu_{c}$ can then be expressed using the following formulas:

$$
L\left(\mu \leftrightarrow \mu_{o} \vee \mu_{c}\right) \quad L\left(\mu_{o} \wedge \mu_{C} \leftrightarrow \perp\right)
$$

Such constraints are Boolean constraints. For example, a simple Boolean lattice validating the two formulas concerning moras above is $\left(\left\{\mu, \mu_{o}, \mu_{c}, \perp\right\} ; \mu_{o} \sqcap \mu_{c}=\perp\right.$, $\left.\mu_{o} \sqcup \mu_{c}=\mu\right\rangle$. This is depicted as a diagram as follows:

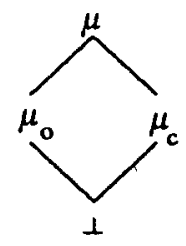

Each element of $\mathbf{X}$ appears as a node in the diagram. The join (ப) of two sorts $p$ and $q$ is the unique sort found by following lines upwards from $p$ and $q$ until they first connect, and conversely for the meet $(\Pi)$. For convenience, constraints on node classifications will be depicted using lattice diagrams of the above form. Trading on the fact that $\mathcal{L}$ contains propositional calculus, Boolean constraints can be uniformly expressed in $\mathcal{L}$ as follows:

(i) $p \sqcap q=r$ becomes $L(p \wedge q \leftrightarrow r)$

(ii) $p \sqcup q=r$ becomes $L(p \vee q \leftrightarrow r)$

Appropriateness Constraints. As we shall see, the hierarchical prosodic structures of phonology are highly constrained. For example, syllables dominate moras and not the other way around, and so a structure where there is a mora dominating a syllable is ill-formed. Following (Bird 1990), we express these restrictions on dominance by augmenting the sort lattice with a binary' 'appropriateness' relation $A$, represented graphically using arrows. We can express in $\mathcal{L}$ the constraints captured by such appropriateness graphs. For example, $L(p \rightarrow \neg \diamond q)$ expresses the fact that a node with sort $p$ cannot dominate a node with sort $q$. We can also use $L$ to express stronger constraints. For example, $L(p \rightarrow \diamond q)$ expresses the fact that a node of sort $p$ must dominate at least one node of sort $q$. In short, the $\diamond$ operator allows us to express graphical constraints.

\section{A THEORY OF PHONOLOGY}

A phonological theory is a collection of generalizations expressed in a language of the above logic. We choose as our language $X=\sigma, \sigma_{h}, \sigma_{l}, \sigma_{o}, \sigma_{c,} \sigma_{h o}, \sigma_{h c}, \sigma_{l o}, \sigma_{l c}, \mu$, $\mu_{o}, \mu_{c}, \pi, \pi_{c}, \pi_{v}, b, d, h, j, k, n, r, s, t, w, ?, a, u, l$. The nine $\sigma$ symbols are for the classification of syllables into heavy us. light and open vs. closed and their various cross classifications. We have already been introduced to $\mu, \mu_{o}$ and $\mu_{c}$, for moras, onsets and codas respectively. The remaining symbols are classifications of segments $(\pi)$, firstly into vowels $\left(\pi_{v}\right)$ vs. consonants $\left(\pi_{c}\right)$, and then into the individual segments themselves (in boldface). This classification is depicted in (2) below.

Syllable Structure. Phonological representations for sta, tat, taat and ast are given in $(1)^{5}$.
(1)

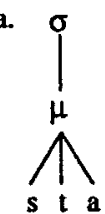

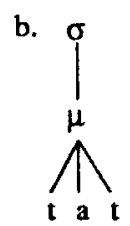

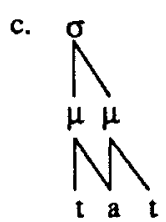

We can describe these pictures using formulas from $L$. For example, (1c) is described by the formula:

$$
\sigma \wedge\langle\mu\rangle(F j \wedge\langle\pi\rangle \mathbf{t} \wedge\langle\pi\rangle(\mathbf{a} \wedge i)) \wedge\langle\mu\rangle(j \wedge\langle\pi\rangle i \wedge\langle\pi) \mathbf{t})
$$

It is possible to use formulas from $\mathcal{L}$ to describe ill-formed syllable structures. We shall rule these out by stating in $\mathcal{L}$ our empirical generalizations. We begin by specifying (i) the relationship between the sorts (i.e. the set $X$ ) using a sort lattice and (ii) how the sorts interact with dominance using an appropriateness relation. We then express in $\mathcal{L}$ the constraints graphically represented in the appropriateness graph in (2).

(2)

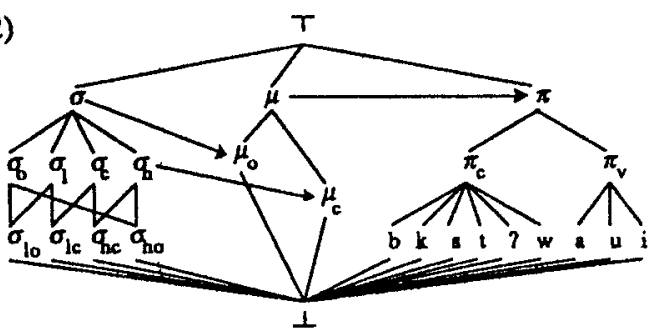

The arrows may be glossed as follows: (i) all syllables must dominate an onset mora, (ii) heavy syllables must dominate a coda mora and (iii) all moras must dominate a segment. The fact that potential arrows are absent also encodes constraints. For example: (i) syllables, moras and segments alike cannot dominate syllables and (ii) light syllables do not have coda moras. Constraints concerning the number of nodes of sort $p$ that a node of sort $q$ can dominate, and constraints concerning temporal organization cannot be stated in this graphical style. Nevertheless, they can be expressed in $L$ as follows.

\section{(A1) $L\left(\left\langle\mu_{0}\right\rangle \phi \rightarrow\left[\mu_{0}\right] \phi\right)$. Onsets are unique.}

\footnotetext{
${ }^{5}$ See (Bird 1990) for arguments justifying this view of syllable structure. Moras are traditionally employed in the representation of syllable weight: a syllable with two moras is heavy, and a syllable with one mora is light.
} 
(A2) $L\left(\left\langle\mu_{c}\right\rangle \phi \rightarrow\left[\mu_{c}\right] \phi\right)$. Codas are unique.

(T7) $L\left(\left\langle\mu_{c}\right\rangle \phi \rightarrow\left\langle\mu_{o}\right\rangle F \phi\right)$. Onsets precede codas.

(T8) $L\left(\sigma_{o} \rightarrow\left\langle\mu, \pi_{v}\right\rangle \phi \wedge\left[\mu, \pi_{c}\right] F \phi\right)$. An open syllable ends with a vowel.

(T9) $L\left(\sigma \rightarrow \neg\left(\left\langle\mu, \pi_{v}\right\rangle F \phi\right.\right.$ $\left.\left.\wedge\left\langle\mu, \pi_{c}\right\rangle(\phi \wedge F \psi) \wedge\left\langle\mu, \pi_{v}\right\rangle \psi\right)\right)$.

The vowels of a syllable must form an unbroken sequence.

(T10) $L\left(\sigma_{h} \rightarrow \neg\left\langle\mu_{o}\right\rangle\left(\left\langle\pi_{v}\right\rangle F \phi \wedge\left\langle\pi_{c}\right\rangle \phi\right)\right)$.

In a heavy syllable, the onset cannot end with a consonant.

We can express the constraint that two syllables cannot share a mora as follows.

(A3) $L(i \wedge \sigma \wedge\langle\mu\rangle j \rightarrow \neg M(\neg i \wedge\langle\mu\rangle j))$.

Two syllables cannot share a mora.

An interesting alternative is $t 0$ add an operator $\diamond^{-1}$ that looks backwards along the dominance relation ${ }^{6}$. The constraint that two syllables cannot share a mora could then be written $L\left(\mu \wedge\langle\sigma\rangle^{-1} \phi \rightarrow[\sigma]^{-1} \phi\right)$. There are further phonological phenomena which suggest that this may be an interesting extension of $\mathcal{L}$ to explore. For example, the requirement that all moras and segments must be linked to the hierarchical structure (prosodic licensing) may be expressed thus: $L\left((\mu \vee \pi) \rightarrow \diamond^{-1} \mathrm{~T}\right)$.

Partiality. Crucially for the analysis of Arabic, it is possible to have a formula which describes more than one diagram. Consider the formula $M\left(\sigma \wedge\langle\mu, \pi\rangle\left(t \wedge F_{i}\right)\right) \wedge$ $M(\sigma \wedge\langle\mu, \pi)(\mathrm{a} \wedge i))$, which may be glossed there is a syllable which dominates a $t$, and a syllable which dominates an $a$, and the $t$ is before the $a^{\prime}$. This formula describes the three diagrams in (3) equally well:

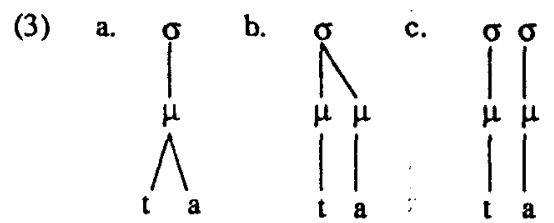

If a level of hierarchical structure higher than the syllable was employed, then it would not be necessary to use the $M$ operator and we could write: $\langle\sigma, \mu, \pi)(t \wedge F i) \wedge$ $\langle\sigma, \mu, \pi\rangle(\mathbf{a} \wedge i)$.

\section{ARABIC VERB MORPHOLOGY}

In the Semitic languages, individual morphemes are often not manifested as contiguious strings of sẹgments. A morphologically complex form must be expressed as the intercalation of its component morphemes. An example of this phenomena is illustrated in Figure 1 for the perfective active? ${ }^{7}$. Consider the form kattab in the second row. Its particular arrangement of four consonants and two

\footnotetext{
${ }^{6}$ That is, $\diamond^{-1}$ is to $\diamond$ as $P$ is to $F$.

7 Note that these are uninflected forms. Some forms are actually non-existent (for semantic reasons); these are indicated by a dash. However, this is unimportant since the present interest is in phonological structure and in potential forms.
}

\begin{tabular}{|c|c|c|}
\hline Conj & Verb Form & Gloss \\
\hline I & katab & wrote \\
\hline II & kattab & caused to write \\
\hline III & kaatab & corresponded \\
\hline IV & ?aktab & diclated \\
\hline V & takattab & - \\
\hline VI & takaatab & kept up a correspondence \\
\hline VII & nkatab & subscribed \\
\hline VIII & ktatab & copied \\
\hline IX & ktabab & - \\
\hline $\mathbf{X}$ & staktab & had a copy made \\
\hline $\mathbf{X I}$ & ktaabab & - \\
\hline XII & ktawtab & - \\
\hline XIII & ktawwwab & - \\
\hline XIV & ktañbab & - \\
\hline XV & ktanbay & - \\
\hline II & dahraj & rolled \\
\hline V & țadaḥraj & caused to roll \\
\hline$X I$ & dharjaj & - \\
\hline XIV & dhanaraj & - \\
\hline
\end{tabular}

Figure 1: Arabic Data based on (McCarthy 1981)

vowels identifies it with the second conjugation. Certain forms have additional affixes which are underlined in the above table. In what follows, we make a number of observations about the patterning of consonants in the above forms, showing how these observations can be stated in $L$. Arabic Syllable Structure. It is now widely recognized amongst phonologists that an analysis of Arabic phonology must pay close attention to syllable structure ${ }^{8}$. From the range of syllable structure possibilities we saw in (1), only the following three kinds are permitted in Arabic.
(4) a

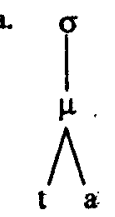

b.

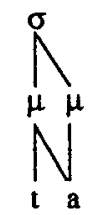

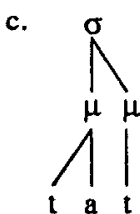

The following generalizations can be made about Arabic syllable structure.

(A4) $L\left(\sigma_{c} \rightarrow \sigma_{h}\right)$. Closed syllables are heavy.

(A5) $L\left(\left\langle\pi_{c}\right\rangle \phi \rightarrow\left[\pi_{c}\right] \phi\right)$.

There is a maximum of one consonant per node.

(A6) $L\left(\left(\pi_{v}\right) \phi \rightarrow\left[\pi_{v}\right] \phi\right)$.

There is a maximum of one vowel per node.

(A7) $L\left(\mu_{c} \wedge(\pi) \phi \rightarrow[\pi] \phi\right)$.

There is a maximum of one segment per coda.

(A8) $L\left(\left\langle\mu, \pi_{v}\right\rangle \phi \rightarrow\left[\mu, \pi_{\nu}\right] \phi\right)$.

There is a maximum of one vowel per syllable.

\footnotetext{
${ }^{8}$ The approaches to Arabic phonology presented by Kay (1987) and Gibbon (1990)-while addressing important computational issues-fail to represent the hierarchical organization of phonological structures.
} 
(T11) $L\left(\mu_{0} \rightarrow\left\langle\pi_{c}\right\rangle F \phi \wedge\left\langle\pi_{\nu}\right\rangle \phi\right)$.

Onsets must have a consonant and a vowel, in that order.

There are certain phonological phenomena which appear to move us beyond the bounds of $L$ : the need to specify defaults. Phonologists often employ default consonants and vowels, which appear when the consonant or vowel positions in syllable structures have not been filled. In Arabic, the defanlt consonant is ? (the glottal stop) and the default vowel is a. The default consonant only appears word initially. There are two ways we can treat such defaults. First, we can regard them as instructions on how one ought to 'compute' with $\mathcal{L}$. That is, we regard them as instructions to attempt to build certain preferred models. Alternatively, we could combine $L$ with a default logic.

\section{MORPHOLOGICAL COMBINATION}

Consider the forms kattab and dahraj. Both consist of two closed syllables. This observation is expressed below.

(II) $M\left(\sigma_{c} \wedge i \wedge F j\right) \wedge M\left(\sigma_{c} \wedge j\right) \wedge L(\sigma \rightarrow i \vee j)$

Similarly, the two consonantisms can be represented as follows. (Note that $i_{1}, i_{2}$ and $i_{3}$ are introduced in the (KTB) formula as labels of syllable nodes; these labels will be referred to in the subsequent discussion.)

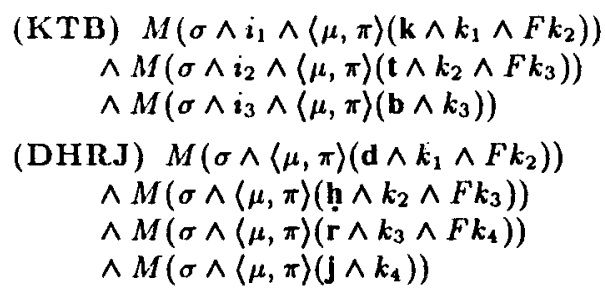

To derive kattab, we simply form (II) $\wedge$ (KTB). The final conjunct of (II) requires that there be only two syllables. Consequently, each syllable mentioned in (KTB) has to be identified with $i$ or $j$. There are eight possibilities, which fall into three groups. In what follows, $i \approx j$ is shorthand for $L(i \leftrightarrow j)$, i.e. $L$ is rich enough to support a form of equational reasoning ${ }^{9}$.

(i) $i_{1} \approx i_{2} \approx i_{3} \approx i$ or $i_{1} \approx i_{2} \approx i_{3} \approx j$. This would require a syllable to dominate three distinct consonants. However, from (A1), (A2) and (A5), Arabic syllables contain a maximum of two consonants.

(ii) $i_{1} \approx i_{3} \approx i, i_{2} \approx j ; i_{2} \approx i_{3} \approx i, i_{1} \approx j ; i_{2} \approx i, i_{1} \approx$ $i_{3} \approx j$; or $i_{3} \approx i_{1} i_{1} \approx i_{2} \approx j$. In all of these cases, we have the following reductio ad absurdum, for some $k^{\prime} \in\left\{k_{1}, k_{2}, k_{3}\right\}$.

$$
\begin{array}{ll}
M\left(\sigma \wedge\left(\mu, \pi_{c}\right\rangle k^{\prime} \wedge F j\right) \wedge M\left(\sigma \wedge j \wedge\left\langle\mu, \pi_{c}\right\rangle F k^{\prime}\right) \\
M\left(O k^{\prime} \wedge F j\right) \wedge M\left(j \wedge O F k^{\prime}\right) & (D 1) \\
M\left(O k^{\prime} \wedge F O F k^{\prime}\right) & (N O M) \\
M\left(O k^{\prime} \wedge F k^{\prime}\right) & (T 5) \\
M\left(O k^{\prime} \wedge \neg O k^{\prime}\right) & (T 4)
\end{array}
$$$$
\perp
$$

${ }^{9}$ Other useful 'macros' are $i \prec j$, which expands to $L(i \rightarrow$ $F j)$, and $i \circ j$, which expands to $L\left(i \rightarrow O_{j}\right)$. (iii) $i_{1} \approx i_{2} \approx i, i_{3} \approx j$ or $i_{1} \approx i, i_{2} \approx i_{3} \approx j$. It follows from the above default stipulations that two of the four consonants of (II) must be identical. By a similar process to that used in (ii) above, we can show that the coda consonant of $i$ is identical to the onset consonant of $j$. The result is shown in ( $5 \mathrm{a}$ ).
(5)

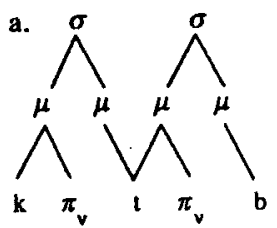

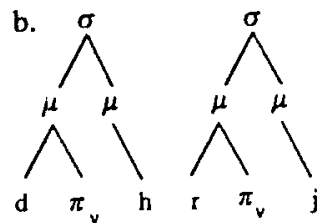

The case of (II) $\wedge$ (DHRJ) is depicted in (5b). The four consonants of (DHRJ) satisfy the requirements of the second conjugation template (II) without the need for reentrancy.

\section{OTHER PHENOMENA}

Consonant Doubling. In conjugations IX, XI, XII, XIV and QIV there is a non-geminate doubling of consonants. In the exceedingly rare XII, the second consonant $(t)$ is doubled. In all the other cases, the final consonant is doubled. The most direct solution is to posit a lexical rule which freely applies to consonantisms, doubling their final consonant. For example, the rule would take the (KTB) form provided above and produce:

(KTB') $\left.\left.M\left(\langle\mu, \pi\rangle \wedge \mathrm{k} \wedge F k_{1}\right)\right) \wedge M\left(\langle\mu, \pi\rangle \wedge t \wedge k_{1} \wedge F k_{2}\right)\right)$ $\left.\left.\wedge M\left((\mu, \pi) \wedge \mathrm{b} \wedge k_{2} \wedge F k_{3}\right)\right) \wedge M\left((\mu, \pi\rangle \wedge \mathrm{b} \wedge k_{3}\right)\right)$

It would be necessary to prevent this extended form from being used in conjugations $I$ and $V$, since the patterns katbab and takatbab are unattested.

The Reflexive Affix. Conjugations V, VI and VIII are marked by the.presence of a reflexive affix $t$. Rather than viewing these conjugations as basic (as Figure 1 implies), we can treat them as having been derived from conjugations II, III and I respectively. As this affix always appears as the onset of a light syllable, we shall represent it thus: $M\left(\sigma_{l} \wedge\langle\mu, \pi\rangle t\right)$. This morpheme will actually be expressed lexically as a function from conjugations to conjugations which attaches a syllable of the above form to the existing phonological material of a conjugation. The affix must be ordered relative to the other syllables. A constraint encoding the observation that all conjugations end in a closed syllable would prevent the affix from being a suffix. The fact that the affix is a light syllable correctly rules out ktattab (V) and ktaatab (VI), leaving only the attested forms for (V) and (VI). A constraint which prevented the first two syllables of a conjugation both being light (cf. McCarthy 1981:387), easily expressed in $L$, would rule out takatab (VII) leaving only the attested form for (VIII).

Extrametricallty. Above we specified conjugation II as having a closed final syllable. Looking back at Figure 1, it would appear as if all conjugations end in a closed syllable. However, a study of the inflected forms reveals that this is not the case. For example, the third person plural of kattab is kattabu. From (T11), the b must be syllabified with the vowel to its right. However, the first person plural 
is kattabna, and the $b$ is syllabified with the vowel to its left. Similarly, the s of staktab is not part of the syllable tak. It is actually the coda of a previous syllable. In order to pronounce this form, ?I is prefixed, producing ?istaktab.

Therefore, the conjugations are not merely sequences of complete syllable templates, but rather they are sequences bounded by unsyllabified (or extrametrical) consonants. The definition of II should therefore be modified to be $M \sigma_{c} \wedge$ $M\left(\sigma \wedge\left\langle\mu_{0}\right\rangle\right) \wedge M(\sigma \wedge\langle\mu\rangle){ }^{10}$. This is intended to leave open the possibility for the final consonant to be syllabified with the second syllable or with the third syllable, while simultaneously requiring it to ultimately be syllabified somewhere.

\section{CONCLUSION}

In this article we have presented an application of interval based tense logic to 'non-linear' phonology (specifically, 'autosegmental' phonology, Goldsmith 1990), and exemplified it using data from Arabic (McCarthy 1981). The chief difference between this view of phonology and its purely segmental predecessors is its use of overlapping intervals of time.

As argued in (Bird 1990), the three primitives: dominance, precedence and overlap are sufficient to represent hierarchically and temporally organized phonological structure. Here we have taken a standard language of interval based tense logic, augmented it with an extra operator $\diamond$ to express phonological dominance, and employed nominals to enable us to label nodes. A universal theory of syllable structure was expressed in $\mathcal{L}$, to which further generalizations were added for Arabic. We then showed how so-called 'non-concatenative' morphology might be treated, and indicated how the phonological notions of extrametricality and licensing were cashed out. The analysis of Arabic did not require recourse to separate consonant and vowel tiers (following Hudson 1986). Rather, consonantisms (and vocalisms) are simply partially specified phonological structures which may be combined using logical conjunction. Even so, the exemplification given was for but a tiny fragment of Arabic phonology and much work is still to be done. For example, nothing was said about the vocalisms.

The move from linear to non-linear phonology parallels the move from a purely Priorean tense logic in $F$ and $P$ to interval based systems in $F, P$ and $O$. The view of phonology emerging from the present study is significantly more formal than many of its contemporaries (cf. Bird \& Ladd 1991), and suggests that enhanced modal formalisms may provide a natural foundation for rigorous phonological theorizing.

Finally, there would seem to be good reasons for being confident that complex phonological descriptions can now be fully incorporated into feature structure based grammar frameworks. Reape's (1991) logical foundation for these frameworks and the phonological arguments in favour of adopting feature structures (Hayes 1990, Bird 1991) are but two parts of the one story.

\footnotetext{
${ }^{10}$ Note that the exhaustiveness condition $L(\sigma \rightarrow i \vee j)$ and the sequencing constraints in the earlier version of (II) must be expressed here also. They are omitted for the sake of readability.
}

\section{REFERENCES}

Bach, E. (1983). On the relationship between word-grammar and phrase-grammar. Natural Language and Linguistic Theory 1, 65-89.

van Benthem, J. (1983). The Logic of Time. Dordrecht: Reidel.

Bird, S. (1990). Constraint-Based Phonology. Ph.D. Thesis. Edinburgh University.

Bird, S. (1991). Feature structures and indices. Phonology 8(1).

Bird, S. \& E. Klein. (1990). Phonological Events. Journal of Linguistics 26, 33-56.

Bird, S. \& D. R. Ladd. (1991). Presenting Autosegmental Phonology. Journal of Linguistics 27(1).

Blackbum, P. (1989). Nominal Tense Logic. Edinburgh University. CCS/RP-40. To appear in P. Petkov (ed.) Pro. ceedings of the Kleene ' 90 Conference. Springer.

Blackburn, P. (1990). Nominal Tense Logic and other Sorted Intensional Frameworks. Ph.D. Thesis. Edinburgh University.

Gargov, G., S. Passy \& T. Tinchev. (1987). Modal environment for Boolean speculations. In D. Skordev (ed.) Mathematical Logic and its Applications. Plenum Press.

Gargov, G. \& V. Goranko. (1989). Modal Logic with Names I. To appear. Bulgarian Academy of Sciences.

Gibbon, D. (1990). Prosodic association by template inheritance. International Workshop on Inheritance and Natural Language Processing. Tilburg.

Goldsmith, J. (1990). Autosegmental and Metrical Phonology. Oxford: Blackwell.

Hayes, B. (1990). Diphthongization and coindexing. Phonology 7, 31-71.

Hoeksema, J. and R. Janda. (1988). Implications of process morphology for categorial grammar. In Oehrle et al.

Hudson, G. (1986). Arabic root and pattern morphology without tiers. Journal of Linguistics 22, 85-122.

Kay, Martin. (1987). Nonconcatenative finite-state morphology. Proceedings of the 3rd EACL. 2-10.

McCarthy, J. (1981). A prosodic theory of nonconcatenative morphology. Linguistic Inquiry 12, 373-413.

Oehrle, R., E. Bach \& D. Wheeler. (eds.) (1988). Categorial Grammars and Natural Language Structures. Reidel.

Reape, M. (1991). A Formal Theory of Word Order: A Case Study in Germanic. Ph.D. Thesis. Edinburgh University.

Rounds, W. \& A. Manaster-Ramer. (1987). A logical version of functional grammar. Proceedings of the 25 th Annual Meeting of the ACL. 89-96.

Wheeler, D. (1988). Consequences of some categorially motivated phonological assumptions. In Oehrle et al. 